\title{
As we cannot afford to renounce to new generation echocardiography in systemic amyloidosis
}

\author{
Francesco Cappelli ${ }^{1,2}$, Stefano Perlini ${ }^{3}$, Paola Attanà ${ }^{4}$, Gabriele Ciuti ${ }^{5}$, Francesco Salinaro ${ }^{3}$, and Federico Perfetto ${ }^{2}$ \\ ${ }^{1}$ Intensive Cardiac Care Unit, Heart and Vessel Department, University of Florence, Florence, Italy, ${ }^{2}$ Regional Amyloid Center, AOU Careggi, Florence, \\ Italy, ${ }^{3}$ Department of Internal Medicine, Fondazione IRCCS Policlinico San Matteo and University of Pavia, Pavia, Italy, ${ }^{4}$ Florence Heart and Vessels \\ Department, University of Florence, Florence, Italy, and ${ }^{5}$ Department of Internal Medicine, University of Florence, Florence, Italy
}

It was with great interest that we read the recent report by Granstam et al. regarding multimodal characterization of amyloid patients [1]. In their article, the authors summarized the major characteristics of cardiac amyloidosis patients using ECG, standard echocardiography and right heart catheterization parameters.

Indeed two-dimensional (2D), M-mode, and Doppler echocardiography still play a major role in the assessment of amyloid deposition in the heart. However, diagnosis of cardiac amyloidosis merely based on these conventional ultrasonographic techniques is only possible when the disease has already reached a relatively advanced stage, where almost irreversible functional and structural myocardial changes have occurred [2]. According to the consensus criteria of the International Society of Amyloidosis, diagnosis should also be based on the elevation of cardiac biomarkers [3].

As to the imaging techniques, in patient with systemic amyloidosis tissue Doppler (TDI) derived strain and strain rate could assess regional myocardial deformation by detecting left ventricular (LV) longitudinal systolic function abnormalities before LV wall thickness increase and symptoms onset [4].

Moreover, 2D speckle-tracking echocardiography has nowadays enabled the assessment of a particular behavior of LV motion that can be defined as twisting/untwisting. Porciani and coworkers demonstrated that LV twisting and untwisting motions are increased in patients with $\mathrm{AL}$ amyloidosis and no evidence of cardiac involvement, while these motions are reduced in patients with evident amyloidosis cardiac involvement $[5,6]$. This finding suggests that early in the disease, impaired LV relaxation induces a compensatory mechanism based on LV twisting/untwisting motion increase. Such a peculiar behavior that can be detected only by $2 \mathrm{D}$ speckle strain echocardiography, can be useful in diagnosing early cardiac involvement in patient with systemic amyloidosis.

Address for correspondence: Francesco Cappelli, Intensive Cardiac Care Unit, Azienda Ospedaliero Universitaria Careggi, Largo Brambilla 3, 50127, Florence, Italy. Tel: +39557947514. Fax: +39557947706. E-mail: cappellifrancesco@inwind.it
Furthermore, recent reports have demonstrated as both TDI derived strain and 2D speckle-tracking echocardiography can be usefully applied to right ventricular (RV) evaluation in patient with amyloidosis. Bellavia et al. showed that patients with AL amyloidosis and apparently normal 2D and Doppler echocardiography results may present an early impairment of RV longitudinal systolic function, primarily detectable by TDI systolic strain of the basal segment of the RV free wall [7].

In the same line, our group demonstrated that in similar patients, a mean RV longitudinal 2D speckle-tracking strain less negative than $-17 \%$ identifies a cohort of patients with marked RV dysfunction and a high risk of death. In addition, at multivariate analysis RV longitudinal strain was the only echocardiographic predictor of prognosis [8]. This adds on a previous report by Ghio and coworkers [9] on tricuspid annular plane systolic excursion (TAPSE) as a marker of RV systolic dysfunction. In our opinion these studies strongly demonstrate that by these advanced techniques, early functional impairment in cardiac amyloidosis may be detectable when the results of standard echocardiography are still normal or inconclusive.

Furthermore, if a diagnosis of systemic amyloidosis has already been made on the setting of haematological, nephrological or neurological impairment, the role of the cardiological evaluation becomes essential in confirming or excluding a possible cardiac involvement, which has a profound impact on patient's prognosis as well as on the therapeutical approach. In patients who do not have overt cardiological disease, diagnosis of cardiac amyloid involvement is particularly challenging. Given that early diagnosis of cardiac amyloidosis should be the primary goal of the cardiologist, in such a situation we believe that a comprehensive cardiac amyloidosis evaluation should be integrated by the use of $2 \mathrm{D}$ speckle strain echocardiography or at least by the support of a TDI derived strain imaging assessment. Indeed, the possibility to detect a very carly cardiac involvement with more sensitive diagnostic tools could be very helpful in improving diagnosis, in stratifying prognosis and in guiding the therapeutic strategy in amyloidosis patients. 


\section{Declaration of interest}

The authors report no conflicts of interest. The authors alone are responsible for the content and writing of this article.

\section{References}

1. Granstam SO, Rosengren S, Vedin O, Kero T, Sörensen J, Carlson K, Flachskampf FA, et al. Evaluation of patients with cardiac amyloidosis using echocardiography, ECG and right heart catheterization. Amyloid 2013;20:27-33.

2. Perfetto F, Cappelli F, Bergesio F, Ciuti G, Porciani MC, Padeletti L, Moggi Pignone A. Cardiac amyloidosis: the heart of the matter. Intern Emerg Med. Intern Emerg Med 2013;8:191-203.

3. Gertz MA, Merlini G. Definition of organ involvement and response to treatment in AL amyloidosis: an updated consensus opinion. Amyloid 2010;17:48-9.

4. Srinivasan G, Joseph M, Selvanayagam JB. Recent advances in the imaging assessment of infiltrative cardiomyopathies. Heart 2013; 99:204-13.

5. Porciani MC, Cappelli F, Perfetto F, Ciaccheri M, Castelli G, Ricceri I, Chiostri M, et al. Rotational mechanics of the left ventricle in AL amyloidosis. Echocardiography 2010;27: 1061-8.

6. Cappelli F, Porciani MC, Bergesio F, Perfetto F, De Antoniis F, Cania A, Tronconi F, et al. Characteristics of left ventricular rotational mechanics in patients with systemic amyloidosis, systemic hypertension and normal left ventricular mass. Clin Physiol Funct Imaging 2011;31:159-65.

7. Bellavia D, Pellikka PA, Dispenzieri A, Scott CG, Al-Zahrani GB, Grogan M, Pitrolo F, et al. Comparison of right ventricular longitudinal strain imaging, tricuspid annular plane systolic excursion, and cardiac biomarkers for early diagnosis of cardiac involvement and risk stratification in primary systematic (AL) amyloidosis: a 5-year cohort study. Eur Heart J Cardiovasc Imaging 2012;13:680-9.

8. Cappelli F, Porciani MC, Bergesio F, Perlini S, Attanà P, Moggi Pignone A, Salinaro F, et al. Right ventricular function in AL amyloidosis: characteristics and prognostic implication. Eur Heart J Cardiovasc Imaging 2012;13:416-22.

9. Ghio S, Perlini S, Palladini G, Marsan NA, Faggiano G, Vezzoli M, Klersy C, et al. Importance of the echocardiographic evaluation of right ventricular function in patients with $\mathrm{AL}$ amyloidosis. Eur J Heart Fail 2007;9:808-13. 